\title{
Some problem of centrifugal compressors upgrading
}

\author{
Nikolai Sadovskiy ${ }^{1,}$, Leonid Strizhak ${ }^{1}$, Anatoliy Simonov ${ }^{1}$, and Mikhail Sokolov ${ }^{1}$ \\ ${ }^{1}$ St. Petersburg State Polytechnic University, St. Petersburg, Russia
}

\begin{abstract}
The results of computational investigation of the influence of a gas labyrinth seals, oil end seals with floating rings and oil journal bearings lubricating layer stiffness coefficient on centrifugal compressor rotor critical speed are presented and analysed. The main principles of a method for axial forces acting on centrifugal compressor rotor calculation are listed. The method has been developed at the compressor, vacuum and refrigeration technologies department of SPbSTU. In addition, the estimation of method applicability in engineering calculations is given.
\end{abstract}

\section{Introduction}

At present, there is an intensive modernization of existing natural gas superchargers. The flow parts are changed in order to increase their efficiency. For this purpose, low-pressure impellers (Imp) are often used, i.e. Impellers with smaller (in comparison with conventional Imp) coefficients of theoretical head and, accordingly, with a greater degree of reactivity. In such cases, while maintaining the final parameters of the compressor, it becomes necessary to increase the rotor speed, the length of the Imp diameter $\mathrm{D}_{2}$, or the number of stages.

Reliability of axial forces determination for multistage centrifugal compressors is the key to their reliable and trouble-free operation. Currently, there is no universal method for calculating axial forces for centrifugal compressors. All methods are to some extent "tied" to specific designs of compressor rotors. The development of the proposed method is a continuation of the work aimed at expanding the capabilities of computational methods for determining the axial forces with acceptable accuracy.

The aim of the study was to obtain experimental data for their use in the methods creation for calculating the axial forces of centrifugal stages, taking into account the different nature of the flow in the side gaps of the impeller and the surface roughness.

The objects of the study were five standard sizes of low-flow intermediate stages, which were studied at different Reynolds numbers and changes in some geometric parameters: axial gaps between the disk and the body, different gaps in labyrinth seals, as well as the disks surfaces roughness and the body. Studies were conducted on the closed high-pressure circuit stand of the Compressor Vacuum and Refrigeration Engineering Department. The change in the Reynolds number was made by changing the pressure in the stand. The data obtained were generalized in the form of empirical dependences, which were used to determine the

* Corresponding author: levo-box@mail.ru 
corresponding velocities profiles required to solve the equations describing the gas flow in axial gaps and labyrinth seals.

When calculating the $\mathrm{n}_{\mathrm{cr}}$ rotor of a single-shaft five-stage $\mathrm{CC}$ that compresses hydrogencontaining gas (rotor length $\mathrm{L}=1.605 \mathrm{~m}$, mass $\mathrm{M}_{\mathrm{r}}=237.8 \mathrm{~kg}$, shaft diameter in support bearings $\mathrm{d}_{\mathrm{sb}}=0.07 \mathrm{~m}$, maximum shaft diameter $\mathrm{d}_{\mathrm{s} \cdot \max }=0.174 \mathrm{~m}$, mass of the impellers $\mathrm{M}_{\mathrm{imp}}$ $=13.3 \ldots 11.6 \mathrm{~kg}$ ), with an increase in the coefficient of rigidity of the oil layer in the reference OS from $10^{8}$ to $10^{10} \mathrm{~N} / \mathrm{m}$, the speed of the first harmonic of $\mathrm{n}_{\text {cr }}$ increased almost 1.3 times, the second harmonic - in approximately 1.5 times. Reduction of the stiffness coefficient of the gas layer $\mathrm{kg}_{\mathrm{g}}$ in the end LS of the shaft (from $1.6^{*} 10^{6}$ to $2 * 10^{5} \mathrm{~N} / \mathrm{m}$ ) and in the LS dummis (from $4.6^{*} 10^{6}$ to $5^{*} 10^{5} \mathrm{~N} / \mathrm{m}$ ) with $\mathrm{k}_{\mathrm{m}}$ equal $10^{8} \ldots 10^{10} \mathrm{~N} / \mathrm{m}$ practically does not affect the value $\mathrm{n}_{\mathrm{cr}}$ of the rotor [11-20].

For a rotor of a two-stage centrifugal natural gas fuel $\left(\mathrm{L}=2.35 \mathrm{~m}, \mathrm{M}_{\mathrm{r}}=1038 \mathrm{~kg}, \mathrm{~d}_{\mathrm{s}}=\right.$ $0.125 \mathrm{~m}, \mathrm{~d}_{\mathrm{s} \cdot \max }=0.339 \mathrm{~m}, \mathrm{D}_{2}=0.819 \mathrm{~m}, \mathrm{k}_{\mathrm{g}}$ in the end rolls of the shaft 0.44106 and 1.74106 $\mathrm{N} / \mathrm{m}$ ) with a change in $\mathrm{k}_{\mathrm{m}}$ in the reference OS from $10^{8}$ to $10^{10} \mathrm{~N} / \mathrm{m} \mathrm{n}_{\mathrm{cr}}$ the first harmonic increased more than 1.5 times.

At the second stage of computational studies, the ANSYS program was used with a finite element method and the construction of an axisymmetric computational model geometrically closest to the real rotor [2]. The non-axisymmetric Imps are modeled as axisymmetric, with effective rigid and mass properties. Supportive OS slip and OSFR, gas LS - on the covering discs Imp, interstage and dummis sealing were modeled by linear elastic elements with the corresponding rigidity characteristics, which are equal to the real rigidity of the lubricating layer in these elements. In the case of the DGS, they were modeled in the form of point masses.

\section{Methods}

Calculations were carried out for the rotors of two centrifugal superchargers of natural gas (produced by the PAO "NPO named after MV Frunze"): a two-stage jet pump NCV-6.3-56 / $1.7\left(\mathrm{M}_{\mathrm{r}}=390 \mathrm{~kg}, \mathrm{~L}=1,802 \mathrm{~m}\right.$, the length between the supporting bearings is $\mathrm{L}_{\mathrm{b}}=1.44 \mathrm{~m}, \mathrm{~d}_{\mathrm{b}}$ $=0.1 \mathrm{~m}, \mathrm{~d}_{\mathrm{b} \text {. } a x}=0.16 \mathrm{~m}, \mathrm{M}_{\mathrm{imp}} \sim 59$ and $58 \mathrm{~kg}$, dummis mass $\sim 38.5 \mathrm{~kg}$, thrust disk mass and respectively, -10.3 and $17.8 \mathrm{~kg}$ ) and six-stage supercharger NC 16-200/1.7 (L / p = 750 kg, $\mathrm{L}=2.69 \mathrm{~m}, \mathrm{~L}_{\mathrm{b}}=2.1 \mathrm{~m}, \mathrm{M}_{\mathrm{imp}}-43 \ldots 44 \mathrm{~kg}, \mathrm{~d}_{\mathrm{b}}=0.16 \mathrm{~m}$, weight of dummis $-12.8 \mathrm{~kg}$, weight of thrust disc and coupling, respectively, -16 and $49.6 \mathrm{~kg}$ ).

For the initial version of each compressor, the following values of the stiffness coefficients of the lubricating layers in the elements were adopted: end LS- $2 * 10^{5}$, liner LS$1.4 * 10^{6} \ldots 2 * 10^{6} \mathrm{H} / \mathrm{m}$, interstage LS $-3 * 10^{5} \ldots 4,3 * 10^{5} \mathrm{H} / \mathrm{m}$, LS dummies $-1,8^{*} 10^{6} \mathrm{H} /$ M, support OS $-10^{9} \mathrm{~N} / \mathrm{m}$, OSFR $-10^{8} \mathrm{H} / \mathrm{m}$ (that corresponds to $\mathrm{k}_{\mathrm{m}}$ in OSFR of the CC of a high pressure).

Here it should be noted that the effect of the coefficients of damping of lubricating layers in seals and bearing bearings by the value of $n_{c r}$ was not noted by researchers $[3,4]$.

As already indicated, in the course of modernization of the acting $\mathrm{CC}$, the flow part is often replaced, which can be accompanied by a change in the geometric and gas dynamic parameters of the stages. In this case, it is possible to change the axial forces acting on the rotor, and the relative losses to the friction of the Imp disks and leakage in the LS of the covering discs.

Determination of the axial force acting on the rotor of the $\mathrm{CC}$, including when changing the operating mode, is necessary to ensure reliable operation of the machine. As the pressure difference in the Imp increases, the effect on the total axial force acting on the Imp increases the different distribution of pressure along the outer surfaces of the Imp disks due to the difference in the direction of leakage in the axial gaps between the discs and the shell. It is known that the flow of leaks in the axial clearance in the radial direction along the covering 
disk is always directed from the periphery to the center. In the axial gap along the main disk in the intermediate stages - from the center to the periphery, and in the end stages - from the periphery to the center.

The method of calculating axial forces acting on the rotor of the $\mathrm{CC}$ was developed at the Department of the CVRT SPbSPU. It uses experimental data on the distribution of pressures in the axial gaps between the Imp and shell discs obtained at various Reynolds numbers in the CVRT department $[5,6]$.

According to the experimental data, the change in the static pressure in the axial gaps between the Imp and the shell is close to linear, the pressure slightly decreases as the radius decreases. In this case, the gradient of the pressure drop near the covering disk is noticeably larger than that of the main disk.

The developed method of calculation of axial forces takes into account the influence of Reynolds numbers, the radial extension of the axial gaps of the Imp and the change in their radial size, the magnitude of gas leaks and pressure drop in the axial gaps, and the topology of the roughness on the movable and fixed walls of the axial gaps. The calculation technique allows us to determine the axial forces for various layouts of the Imp for rotors of singlecenter CCs [7]. The amount of gas leakage in the axial clearance of the wheels is determined by the joint calculation of the viscous gas flow in the gaps and in the LS on the covering disk and on the shaft beyond the Imp.

To calculate the viscous gas flow in lateral axial gaps of the Imp, an approach [8] is applied in which it is assumed that the flow in the gaps is axisymmetric, the gaps between the discs of the Imps and the walls of the casing are narrow, i.e. their width is much less than the length of the gaps, the boundary layers occupy the entire width of the gaps and are closed in their center along the entire radial extent. To take into account the influence of the roughness of the surfaces of the discs Imp and the walls of the shell, the discrete elements method is used [9]. In this case, the real rough surface consisting of protrusions and depressions is replaced by a surface consisting of smooth sections and roughness elements of a given shape, provided that the coefficients of resistance of both surfaces are equal.

The cluttering coefficients (the coefficients of reducing the volume of a smooth gap) are represented in the radial and circumferential direction in the form

$$
\begin{aligned}
& \beta_{m r}=1-\left(\frac{a_{M r s} * k_{M r s}}{2 S t_{r s}}+\frac{a_{M r o} * k_{M r o}}{2 S t_{r o}}\right) \\
& \beta_{m r}=1-\left(\frac{a_{M r s} * k_{M r s}}{2 S t_{r s}}+\frac{a_{M r o} * k_{M r o}}{2 S t_{r o}}\right)
\end{aligned}
$$

where $a_{M r^{3}}, k_{M r^{3}}, t_{r}^{3}, a_{M u^{3}}, k_{M u^{3}}, t_{u^{3}}, a_{u}$ are the basis, height, step between vertices and angle at the base of equilateral triangles of model roughness in the radial and circumferential directions; $\mathrm{S}$ is the width of the gap (the subscripts $\mathrm{s}$ and o refer to the disk and the case, respectively).

The coefficients of decreasing the area of a smooth surface in the radial and circumferential directions are reduced to the form

$$
\begin{aligned}
& \beta_{\tau r}=1-a_{M r} / t_{r} \\
& \beta_{\tau u}=1-a_{M u} / t_{u} .
\end{aligned}
$$

Calculation of the viscous gas flow was performed by an integral method using the equations of continuity, quantity and moment, the amount of motion of the gas mass for the steady flow. The coefficient of resistance of the elements of the roughness of the surfaces of the discs Imp and the walls of the shell was determined with the help of empirical dependence for two-dimensional poorly-flowing solids [10]; 
When calculating the velocity profiles in the axial gaps, the experimental coefficients were used, the expressions for which were obtained as a result of processing the experimental data of the gas flow studies in the axial gaps of the Imp intermediate stages in the range of numbers $\mathrm{Re}=106 . .108$

\section{Results and Discussion}

Calculation of axial forces is performed for three rotor circuits:

1. A rotor with a one-sided arrangement of the Imp, a dummy and with a bypass line connecting the cavity behind the dummies with the suction chamber of the first stage. In this scheme, the permissible axial force, perceived by the thrust bearing, is ensured by the appropriate selection of the diameter of the dummy;

2. Rotor with the opposite location of the Imp ("back-to-back" scheme), with a stepped LS between the sections and with a bypass line connecting the intermediate chamber in the LS with the suction chamber of the first stage. In this case, the axial force is corrected by selecting the diameters of the stepped inter-sectional LS;

3. Rotor with the opposite location of the Imp, intersectional LS and dummies at the end of the shaft. The intersection seal has no intermediate chamber. The cavity behind the dummies is connected by a bypass line with the suction chamber of the first step. In this scheme, the permissible axial force perceived by the thrust bearing can be achieved by performing an inter-sectional seal step-by-step, as well as by changing the diameter of the dummy.

The axial forces acting on the Imp are found by integrating the elementary forces on the outer surfaces of the wheel disks that arise under the action of variables along the radial pressure in the axial gaps between the discs and the body. The distribution of static radial pressures in the axial gaps of the covering and main disks is different. In the intermediate stages this is due to the opposite direction of the flow in the axial gaps and different boundary conditions. In the end stages, despite the same direction of flow in the axial gaps and the same boundary conditions, the different character of the static pressure distribution along the radius of the covering and main disks is associated with the leakage inequality in the axial gaps of these disks.

The reaction of the inflow into the Imp, that is, the value (here the mass flow through the Imp, $\mathrm{c} 0$ is the axial velocity at the entrance in the Imp), and the axial forces acting on the surface of the sections of the rotors outside the Imp are calculated from known simple expressions.

The results of calculations of axial forces acting on the rotors of the Central Committee for various purposes were compared with the results of calculations of axial forces using the technique used in the design of centrifugal compressors at a number of enterprises (Company "REP Holding", OJSC "Compressor Complex", PAO "Frunze Research and Production Association", etc.). The results of calculations for both methods are in good agreement and are almost close to each other.

The results of calculations in solving the equations were:

1. pressure distribution on both impeller disks between the outer radius of the impeller and the radius of the labyrinth seal;

2. distribution of the velocity circumferential component in the axial clearance and disks friction losses;

3. pressure distribution along the length of the labyrinth seal and the mass flow rate of leak through the labyrinth seal;

4. velocity circumferential component distribution along the length of the labyrinth seal and the friction loss in it. 


\section{Conclusions}

In conclusion, we should again emphasize the importance of reliable determination of the axial forces acting on the rotors of the Central Committee in the process of modernization of these compressors, when the replacement of the stubborn OS with magnetic bearings is carried out. It is known that thrust magnetic bearings allow significantly lower specific pressures than plain bearings, and are very sensitive to changes in axial forces.

This research was supported by Compressor vacuum and refrigeration engineering department administration. We thank our colleagues from Compressor vacuum and refrigeration engineering department, who provided insight and expertise that greatly assisted the research.

\section{References}

1. A. V. Zuev, L. Ya. Strizhak I. A. Tuchina, V. M. Vlasov, V. B. Semenovsky. Calculation of the critical speeds of rotation of rotors of turbomachines. Method, instructions / Comp. St. Petersburg: St. Petersburg State Technical University. 32 pp. (1995)

2. ANSYS. ANSYS User Guide for version 5.0. User guide. Copyright ANSYS, Inc., (1995).

3. F. M. Dimentberg, K. S. Kolesnikov. Vibration in engineering. Directory. T.Z. Oscillations of machines, structures and their elements, Ed. M : Mechanical Engineering, 544 p. (1980).

4. E. A. Manushin, I. G. Surovtsev. Design and calculation for the strength of turbomachines of gas turbine and combined plants: Proc. allowance for stud. machine building. specialist. universities. Under. Ed. N.N. Malinina. M .: Mechanical Engineering, 400 pp. (1990)

5. N. I. Sadovsky, K. P. Seleznev, L.Ya Strizhak. Offshore systems and compressors // Compressor engineering and pneumatics. Pp. 27-35. 4-5 (1994).

6. N. I. Sadovsky, M. H. Nguyen. On the evaluation of disk friction and leaks for centrifugal stages // Compressor engineering and pneumatics. Pp. 28-30. 7. (2005).

7. N. I. Sadovsky, L.Ya. Strizhak. Calculation of the axial force acting on the rotor of a multistage centrifugal compressor: Proc. allowance. St. Petersburg: SPbSTU, 38 p. (1998).

8. G. N. Den. Flow mechanics in centrifugal compressors. L. ^ Machine building, 270 p. (1973).

9. G. Taylor, F. Coleman, D. Hodge. Calculation of turbulent surface friction on a rough wall by the method of discrete elements, Tr. Amer. Society inzh.-fur. Theory of engineering calculations. Pp. 163-170. 2. (1985).

10. M. Sokomoto, A. Taniguchi. Profile resistance of three-dimensional non-flowing bodies located in the turbulent boundary layers / Dr. Amer. Society inzh.-fur. Theoretical bases of engineering calculations. Pp. 148-156. 3. (1982).

11. A. A. Aksenov, A. M. Danilishin, Y. V. Kozhukhov, A. M. Simonov. Numerical simulation of gas-dynamic characteristics 3D semi-open impellers of two-stages of centrifugal compressors. AIP Conference Proceedings 2007, 030025; doi: 10.1063/1.5051886. (2018).

12. Y.V. Kozhukhov, A. M. Danilishin, S. V. Kartashov, A. A. Lebedev, K. G. Malev, Y. R. Mironov. Design optimization opportunity of the end stage output plenum chamber of the centrifugal compressor for gas pumping unit. AIP Conference Proceedings 2007, 030044; doi: 10.1063/1.5051905. (2018). 
13. V. V. Neverov, Y. V. Kozhukhov, A. M. Yablokov, and A. A. Lebedev. The experience in application of methods of computational fluid dynamics in correction of the designed flow path of a two-stage compressor. AIP Conference Proceedings 2007, 030048; doi: 10.1063/1.5051909, (2018).

14. A. M. Danilishin, S. V. Kartashov, Y. V. Kozhukhov and E. G. Kozin. The methodology for the existing complex pneumatic systems efficiency increase with the use of mathematical modeling. IOP Conf. Ser.: Mater. Sci. Eng. 232 012069, (2017).

15. A. A Aksenov, A. M Danilishin, A. M Dubenko and Y. V Kozhukov. Development of the virtual experimental bench on the basis of modernized research centrifugal compressor stage test unit with the 3D impeller. IOP Conf. Ser.: Mater. Sci. Eng. 232 012042, (2017).

16. V. V Neverov, Y. V Kozhukhov, A. M Yablokov, A. A Lebedev. Optimization of a centrifugal compressor impeller using CFD: the choice of simulation model parameters. IOP Conf. Ser.: Mater. Sci. Eng. 232 012037, (2017).

17. A. M Danilishin, Y. V Kozhukhov, V. V Neverov, K. G Malev, Y. R Mironov. The task of validation of gas-dynamic characteristics of a multistage centrifugal compressor for a natural gas booster compressor station. Conference Paper in AIP Conference Proceedings 1876(1):020046 DOI: 10.1063/1.4998866 (2017).

18. A. M Danilishin, Y. V Kozhukhov, V. K. Yun. Multi-objective optimization for impeller shroud contour, width of vane diffuser and number of blades of the centrifugal compressor stage based on the CFD calculation. 9th International Conference on Compressors and Their Systems) (City University London). Programme and Book Abstracts. p. 80. (SCOPUS: 2-s2.0-84941236369), (2015).

19. Y. V. Kozhukhov, L. V. Reshetnikova, V. K. Yun, M. V. Procopovich. Numerical Investigation of Different Radial Inlet Forms for Centrifugal Compressor and Influence of the Deflectors Number by Means of Computational Fluid Dynamics Methods with Computational Model Validation. 9th International Conference on Compressors and Their Systems) (City University London). Programme and Book Abstracts. p. 81. (SCOPUS: 2-s2.0-84941254557), (2015).

20. Y. Boldyrev, A. Rubtsov, Y. Kozhukhov, A. Lebedev, I. Cheglakov, A. Danilishin, Simulation of unsteady processes in turbomachines based on nonlinear harmonic NLHmethod with the use of supercomputers. CEUR Workshop Proceedings., Pages 273-279, 1482 (2015). 